Supporting Information

\title{
Visualizing Tetrahedral Oxyanion Bound in HIV-1 Protease Using Neutrons: Implications for Catalytic Mechanism and Drug Design
}

Mukesh Kumar, ${ }^{1,2}$ Kalyaneswar Mandal, ${ }^{3 \#}$ Matthew P. Blakeley, ${ }^{4}$ Troy Wymore, ${ }^{5}$ Stephen B. H. Kent, ${ }^{3}$

John M. Louis, ${ }^{6}$ Amit Das ${ }^{1,2 *}$ and Andrey Kovalevsky ${ }^{7 *}$

${ }^{1}$ Protein Crystallography Section, Radiation Biology and Health Sciences Division, Bhabha Atomic Research Centre, Trombay, Mumbai 400085, India

${ }^{2}$ Homi Bhabha National Institute, Anushaktinagar, Mumbai 400094, India

${ }^{3}$ Departments of Chemistry, and Biochemistry and Molecular Biology, Institute for Biophysical Dynamics, University of Chicago, Chicago, IL 60637, USA

${ }^{4}$ Large Scale Structures Group, Institut Laue-Langevin, 38000 Grenoble, France

${ }^{5}$ Department of Chemistry, University of Michigan, Ann Arbor, MI 48109, USA

${ }^{6}$ Laboratory of Chemical Physics, National Institute of Diabetes and Digestive and Kidney Diseases, National Institutes of Health, DHHS, Bethesda, MD 20892-0520, USA

${ }^{7}$ Neutron Scattering Division, Oak Ridge National Laboratory, Oak Ridge, TN 37830, USA

\#Present address: TIFR Centre for Interdisciplinary Sciences, Tata Institute of Fundamental Research Hyderabad, Hyderabad 500107, India 


\section{Methods}

QM/MM calculations. Model construction was performed with the program CHARMM version c41b2. ${ }^{1}$ The atomistic model was constructed using the atomic coordinates, including the positions of hydrogen atoms, from the joint $\mathrm{XN}$ structure of $\mathrm{PR}_{\mathrm{TM}} / \mathrm{KVS}-1_{\mathrm{TI}}$ complex. All crystallographic water molecules were retained, and the starting models were initially solvated in a cubic box of water molecules with a minimum distance of $10 \AA$ from the protein to the nearest face of the cell. Counterions $\left(\mathrm{K}^{+}\right.$and $\left.\mathrm{Cl}^{-}\right)$corresponding to a biologically relevant 0.15 mol concentration and an overall electrically neutral model were then added. The initial positions of counterions were assigned using a Monte Carlo procedure that accounts for the electrostatic potential of the surface. The CHARMM27 Force Field ${ }^{2}$ with CMAP $^{3}$ corrections and the TIP3P water model were used to describe the protein and solvent, respectively. Force field parameters for KVS- $1_{\mathrm{TI}}$ were obtained from ParamChem. ${ }^{4}$ The charges were slightly adjusted to conform to the CHARMM convention of making each chemical moiety neutral or integral charge. The system size was 44,386 atoms. Two thousand steps of conjugate gradient energy minimization were performed with a $20.0 \mathrm{kcal} \mathrm{mol}^{-1} \AA^{-2}$ harmonic restraint potential on all protein heavy atoms while the KVS- $1_{\text {TI }}$ molecule was held fixed through all equilibration steps in order to retain the structure obtained from experiment. Molecular dynamics (MD) simulations were performed with the same restraint potential on all protein heavy atoms and heated to $300 \mathrm{~K}$ over $50 \mathrm{ps}$ while rescaling the velocities every 1000 steps ( $1 \mathrm{fs}$ time step) in order to equilibrate the bulk solvent and counterions added to the model. Periodic boundary conditions were applied, and the Particle Mesh Ewald method ( 1 grid point $\left.\AA^{-3}\right)$ was used to describe long-range electrostatic effects. The SHAKE algorithm was used to constrain all bonds to hydrogen. Finally, two thousand additional steps of conjugate gradient energy minimization were then performed. The models were then truncated by retaining a $30 \AA$ sphere of atoms centered around the hydroxyl oxygens of the KVS-1 ${ }_{\mathrm{TI}}$ inhibitor, resulting in the starting models for the QM/MM simulations with 11,141 atoms each and with only Arg141/141' not entirely surrounded by at least one water molecule.

All QM/MM calculations were performed with pDynamo ${ }^{5}$ interfaced with ORCA version 3.0. ${ }^{6}$ The QM region was represented by the residues of Asp25/25' (minus the main chain nitrogen and bonded hydrogen), Thr26/26', Gly27/27' (plus the Ala28 main chain nitrogen and bonded hydrogen), the entire KVS inhibitor molecule plus the "flap" water molecule (for a total of 199 QM atoms). The total charge of the QM region was -1. Hydrogen link atoms were automatically placed $1.0 \AA$ away from atoms that formed the QM/MM boundary by pDnamo. All water molecules 
and protein residues containing an atom greater than $20 \AA$ the hydroxyl oxygen of KVS were held fixed. The initial structures very closely matched the XN structure with the restraints supplied to the structure during the MD simulation.

The geometries were then optimized for 100 steps using the conjugate gradient algorithm with the BLYP ${ }^{7,8}, \mathrm{PBE}^{9}, \mathrm{PBE}^{10,11}$, M06 ${ }^{12}$, M06$2 \mathrm{X}^{13}$, B3PW91 ${ }^{14}$, B3LYP ${ }^{7,8,15}$ and wB97 ${ }^{16,17}$ functional and the Ahlrichs def2-SVPD basis set and Grimme D3 dispersion corrections ${ }^{18}$ with BeckeJohnson damping ${ }^{19}$ for all functionals except PBE0, B3PW91, M06 and M06-2x. The resolution of the identity (RI) or RIJCOSX approximation with the SVP/J Coulomb fitting basis was applied in all cases when pure or hybrid functionals were used, respectively. In addition, the spincomponent-scaled MP2 (SCS-MP2) method ${ }^{20}$ was used for 60 steps. All optimizations achieved an RMS displacement of $0.000195 \AA$ or better at the final iteration. All optimizations gave similar reactant structures (Table S3). 
Table S1. Amino acid sequences for the wild-type PR, $\mathrm{PR}_{\mathrm{TM}}$ and PR20. Amino acids in bold green correspond to the stabilizing substitution mutations to restrict autoproteolysis and cysteine-thiol oxidation. Amino acids in bold blue correspond to drug resistant mutations.

\section{PRwt:}

\begin{tabular}{|c|c|c|c|c|c|c|}
\hline 10 & 20 & 30 & 40 & 50 & 60 & 70 \\
\hline PQITLWKRPL & VTIKIGGQLK & EALLDTGADD & TVIEEMSLPG & RWKPKMIGGI & GGFIKVRQYD & QIIIEIAGHK \\
\hline 80 & 90 & 99 & & & & \\
\hline IGTVLVGPT & PVNIIGRNLL & TQIGATLNF & & & & \\
\hline
\end{tabular}

$\mathrm{PR}_{\mathrm{TM}}: \mathrm{V} 32 \mathrm{I}, \mathrm{I} 47 \mathrm{~V}, \mathrm{~V} 82 \mathrm{I}$

\begin{tabular}{|c|c|c|c|c|c|c|}
\hline 10 & 20 & 30 & 40 & 50 & 60 & 70 \\
\hline PQITLWKRPL & VTIKIGGQLK & EALLDTGADD & TIIEEMSLPG & RWKPKMVGGI & GGFIKVRQYD & QIIIEIAGHK \\
\hline 80 & 90 & 99 & & & & \\
\hline
\end{tabular}

PR20: L10F, I13V, I15V, D30N, V32I, L33F, E35D, M36I, S37N, I47V, I54L, Q58E, I62V, L63P, $A 71 V, I 84 V, N 88 D, L 89 T$, L90M

\begin{tabular}{|c|c|c|c|c|c|c|}
\hline 10 & 20 & 30 & 40 & 50 & 60 & 70 \\
\hline PQITLWKRPF & VTVKVGGQLK & EALLDTGADN & TIFEDINLPG & RWKPKMVGGI & GGFLKVREYD & QVPIEIAGHK \\
\hline 80 & 90 & 99 & & & & \\
\hline IGTVLVGPT & PVNVIGRDTM & TQIGATLNF & & & & \\
\hline
\end{tabular}


Table S2. Crystallographic data collection and refinement statistics.

\begin{tabular}{|c|c|c|c|c|}
\hline & \multicolumn{2}{|c|}{$\mathbf{P R}_{\mathrm{TM}} / \mathrm{KVS}-\mathbf{1}_{\mathrm{TI}}, \mathrm{RT}, \mathrm{PDB}$ ID 6PTP } & \multirow{2}{*}{$\begin{array}{l}\mathrm{PR}_{\mathrm{TM}} / \mathrm{KVS}-1_{\mathrm{TI}}, 100 \mathrm{~K}, \text { PDB ID 6KMP } \\
\text { X-ray }\end{array}$} & \multirow{2}{*}{$\begin{array}{l}\mathrm{PR}_{\mathrm{TM}} / \mathrm{DRV}-\mathbf{1}_{\mathrm{TI}}, \mathrm{RT}, \mathrm{PDB} \text { ID 6PU8 } \\
\text { X-ray }\end{array}$} \\
\hline Data collection: & Neutron & X-ray & & \\
\hline Beamline/Facility & LADI-III/ILL & Rigaku HighFlux HomeLab & Advanced Light Source & Rigaku HighFlux HomeLab \\
\hline Space group & & ${ }_{1} 22_{1}$ & $\mathrm{P} 2{ }_{1} 2{ }_{1} 2$ & $\mathrm{P} 2{ }_{1} 2_{1} 2$ \\
\hline \multicolumn{5}{|l|}{ Cell dimensions: } \\
\hline$a, b, c(\AA)$ & \multirow{2}{*}{\multicolumn{2}{|c|}{$\begin{array}{c}59.50,87.40,46.87 \\
90,90,90\end{array}$}} & $57.88,85.24,46.07$ & $59.63,87.44,46.43$ \\
\hline$\alpha, \beta, \gamma\left({ }^{\circ}\right)$ & & & $90,90,90$ & $90,90,90$ \\
\hline Resolution $(\AA ̊)$ & $40.00-2.20(2.32-2.20)^{*}$ & $40.00-1.85(1.92-1.85)^{*}$ & $31.30-1.31(1.34-1.31)^{*}$ & $40.00-1.80(1.86-1.80)$ \\
\hline No. reflections measured & 29884 & 182163 & 240456 & 88289 \\
\hline No. reflections unique & 9666 (1149) & $20942(2012)$ & $53450(3931)$ & $22743(2253)$ \\
\hline$R_{\text {merge }}$ & $0.169(0.327)$ & $0.049(0.489)$ & $0.089(0.459)$ & $0.037(0.481)$ \\
\hline$C C_{1 / 2}^{21}$ & $0.969(0.787)$ & $0.995(0.678)$ & $0.998(0.806)$ & $0.993(0.774)$ \\
\hline$I / \sigma \mid$ & $7.1(3.4)$ & $25.3(3.0)$ & $28.3(3.5)$ & $29.4(2.2)$ \\
\hline Completeness (\%) & $77.3(64.6)$ & $97.6(96.0)$ & $99.6(99.2)$ & $98.0(99.0)$ \\
\hline Redundancy & $3.1(3.2)$ & $3.9(3.8)$ & $3.4(3.1)$ & $3.9(3.8)$ \\
\hline Refinement: & \multicolumn{2}{|c|}{ Joint XN } & X-ray & X-ray \\
\hline Resolution (neutron, $\AA$ ) & \multicolumn{2}{|c|}{$40-2.20$} & & \\
\hline Resolution (X-ray, ̊̊) & \multicolumn{2}{|c|}{$40-1.85$} & $31.3-1.31$ & $20-1.80$ \\
\hline Data rejection criteria & \multicolumn{2}{|c|}{ no observation \& $|F|=0$} & no observation \& $|F|=0$ & no observation \& $|F|=0$ \\
\hline Sigma cut-off & \multicolumn{2}{|c|}{2.5} & 2.0 & \\
\hline No. reflections (neutron) & \multicolumn{2}{|c|}{8804} & & \\
\hline No. reflections (X-ray) & \multicolumn{2}{|c|}{18847} & 53276 & 21357 \\
\hline$R_{\text {work }} / R_{\text {free }}$ (neutron) & \multicolumn{2}{|c|}{$0.234 / 0.257$} & & \\
\hline$R_{\text {work }} / R_{\text {free }}(\mathrm{X}$-ray) & \multicolumn{2}{|c|}{$0.192 / 0.217$} & $0.158 / 0.188$ & $0.188 / 0.239$ \\
\hline \multicolumn{5}{|l|}{ No. atoms } \\
\hline Protein, including $\mathrm{H}$ and $\mathrm{D}$ & \multicolumn{2}{|c|}{3140} & $\begin{array}{l}1602 \text { (including alternate } \\
\text { conformers) }\end{array}$ & 1514 \\
\hline Ligand & \multicolumn{2}{|c|}{$125\left(\mathrm{KVS}-1_{\mathrm{T} 1}\right.$, including $\mathrm{H}$ and $\left.\mathrm{D}\right)$} & $56\left(\right.$ KVS-1 $\left.1_{\mathrm{TI}}\right)$ & $39\left(\mathrm{DRV}_{\mathrm{TI}}\right)$ \\
\hline Water & \multicolumn{2}{|c|}{$312\left(104 \mathrm{D}_{2} \mathrm{O}\right)$} & $435.5\left(\mathrm{H}_{2} \mathrm{O}\right)$ & 121 \\
\hline \multicolumn{5}{|l|}{$B$-factors } \\
\hline Protein & \multicolumn{2}{|c|}{25.4} & 14.6 & 28.5 \\
\hline Ligand & \multicolumn{2}{|c|}{28.3} & 13.5 & 24.2 \\
\hline Water & \multicolumn{2}{|c|}{44.5} & 32.9 & 41.3 \\
\hline \multicolumn{5}{|l|}{ R.M.S. deviations } \\
\hline Bond lengths $(\AA ̊)$ & \multicolumn{2}{|c|}{0.016} & 0.024 & 0.009 \\
\hline Bond angles $\left({ }^{\circ}\right)$ & \multicolumn{2}{|c|}{1.008} & 2.510 & 1.020 \\
\hline
\end{tabular}

* Values in parentheses are for highest-resolution shell. Data were collected from 1 crystal for each structure. 
Table S3. Distance from the Oxygen atom of Wcat to the Carbon of KVS-1 for the different QM/MM methods

\begin{tabular}{|c|c|}
\hline QM Method of QM/MM calculation & Distance $(\AA)$ from C(KVS-1) to O( $\left.\mathrm{W}_{\text {cat }}\right)$ \\
\hline BLYP-D3 & 2.91 \\
\hline PBE-D3 & 2.90 \\
\hline M06 & 2.58 \\
\hline M06-2X & 2.93 \\
\hline B3LYP-D3 & 3.00 \\
\hline PBE0 & 3.04 \\
\hline wB97x-D3 & 3.04 \\
\hline B3PW91 & 2.60 \\
\hline SCS-MP2 & 2.69 \\
\hline DFTB3 & \\
\hline
\end{tabular}




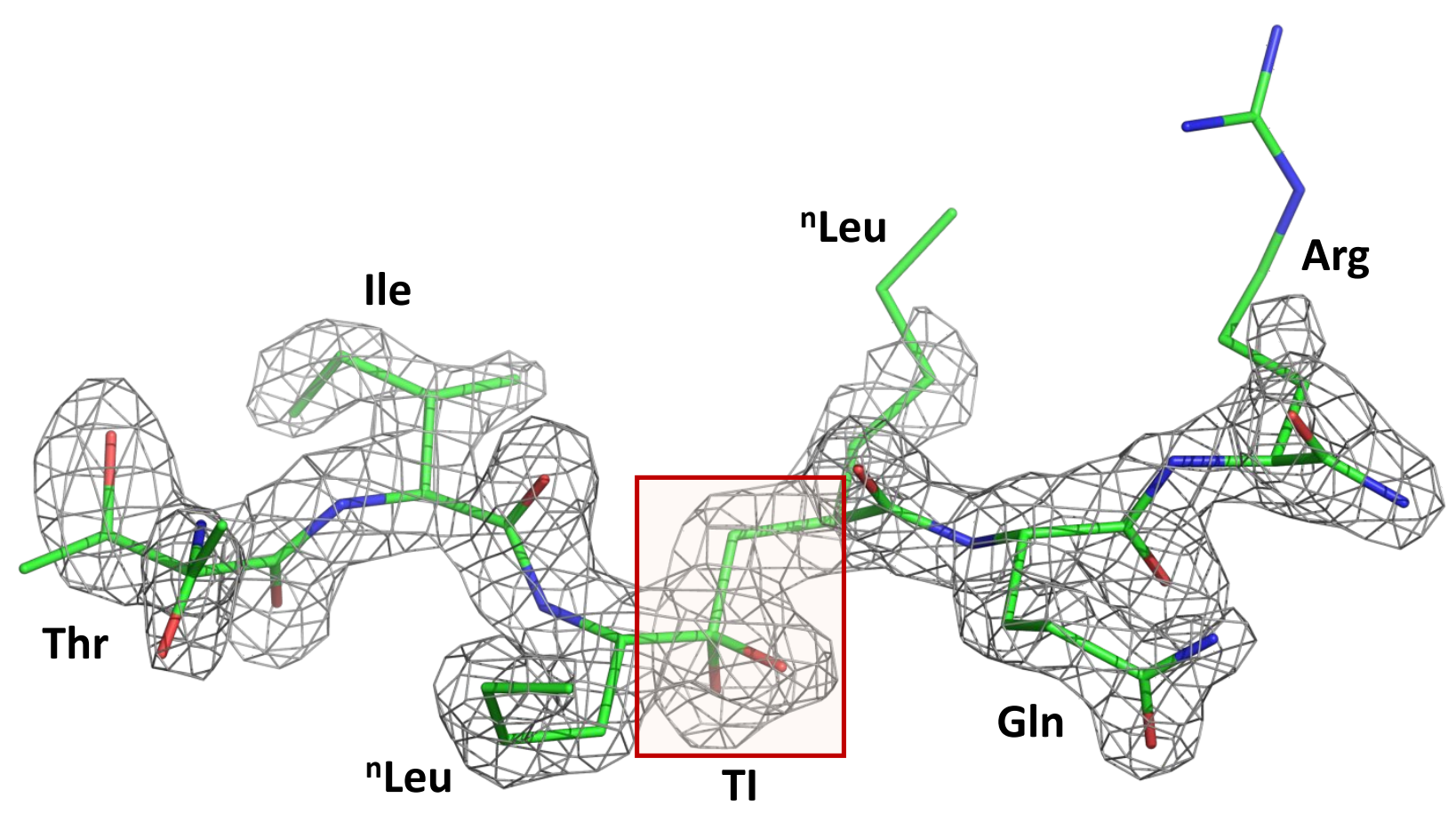

Figure S1. $2 \mathrm{~F}_{\mathrm{O}}-\mathrm{F}_{\mathrm{C}}$ electron density map of KVS-1 $1_{\mathrm{TI}}$ based on the $1.85 \AA$ resolution X-ray diffraction data, contoured at $2 \sigma$ level. Tetrahedral intermediate (TI) moiety created from the initial ketomethylene isostere by a reaction with a water molecule is highlighted by the red box. 


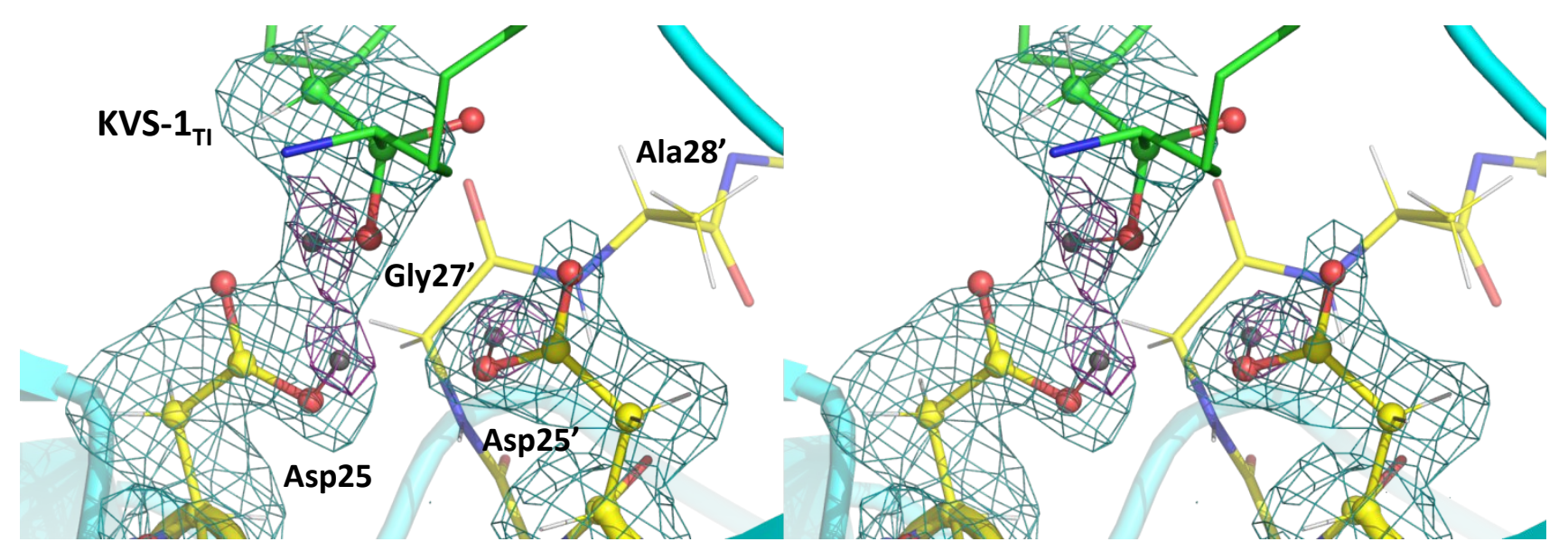

A

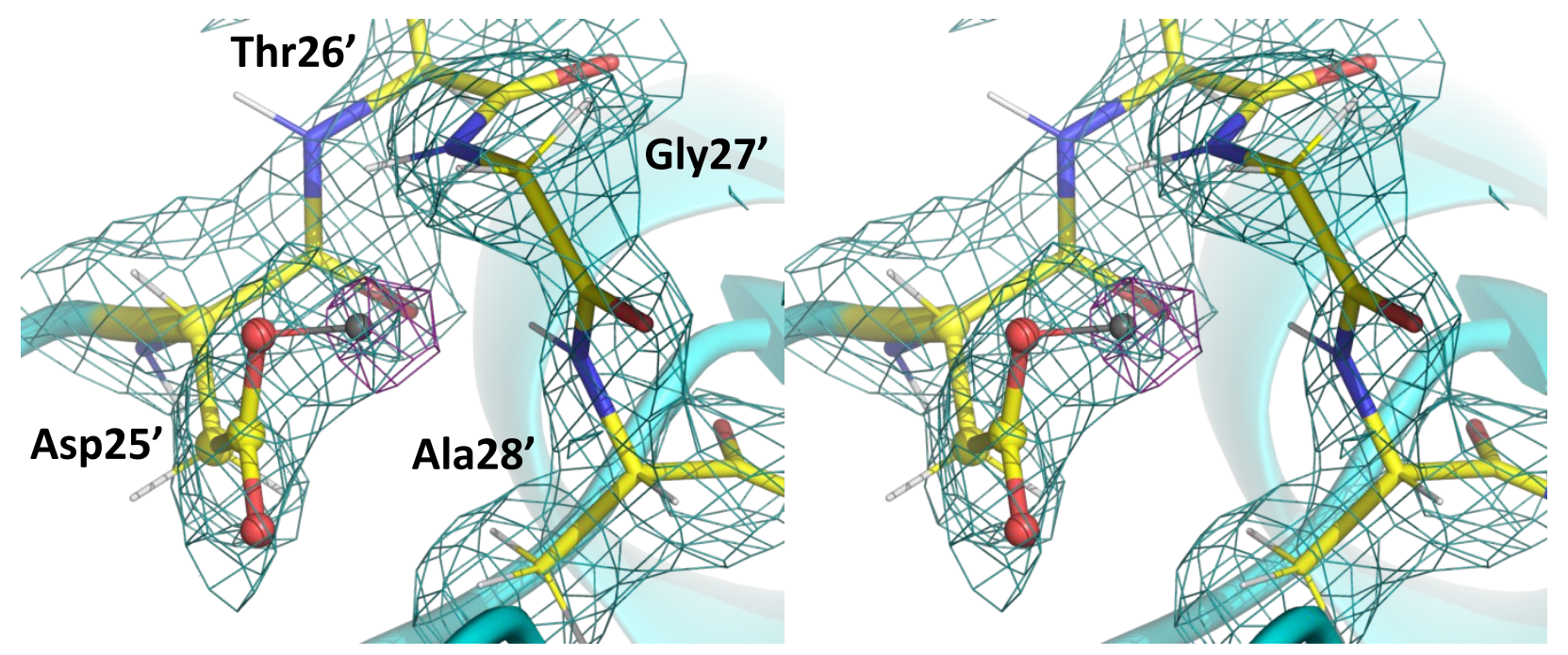

B

Figure S2. A. Figure 4A in crossed-eye stereo mode; B. Figure 4B in crossed-eye stereo mode. 


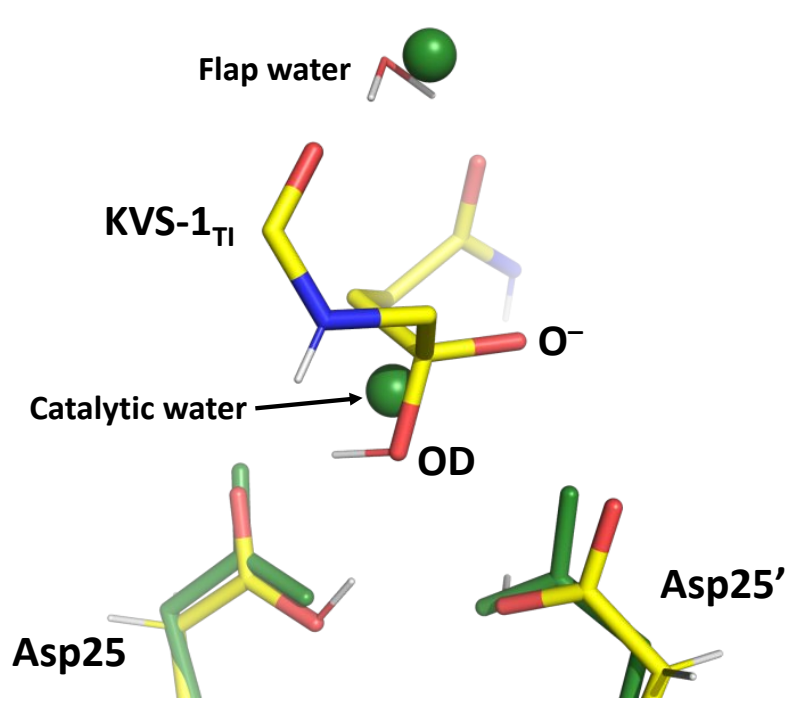

A

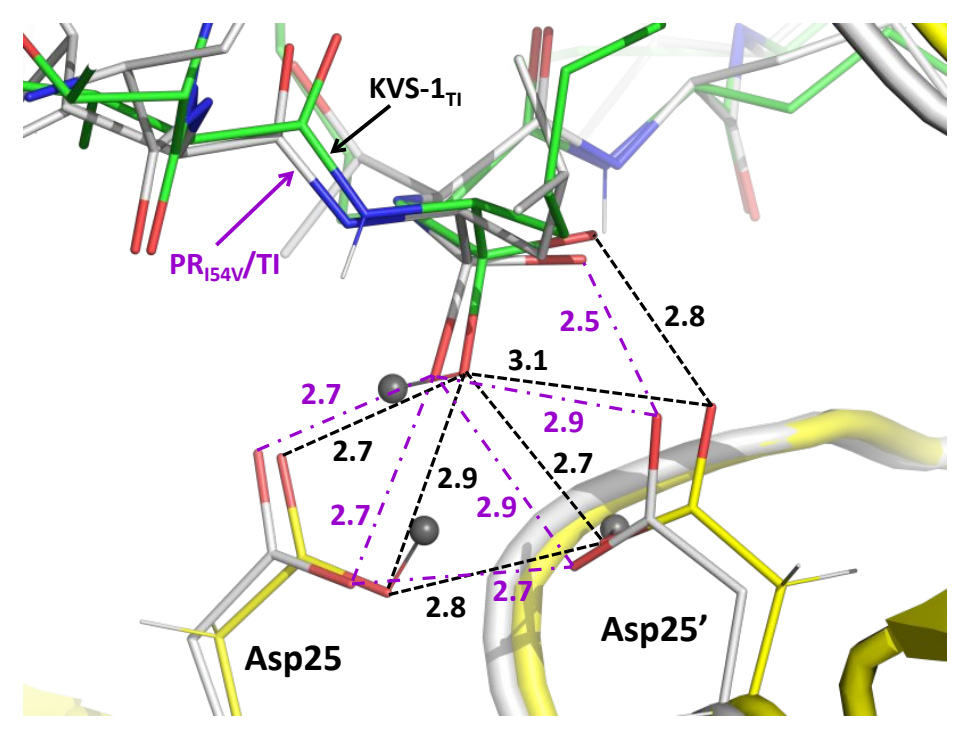

B

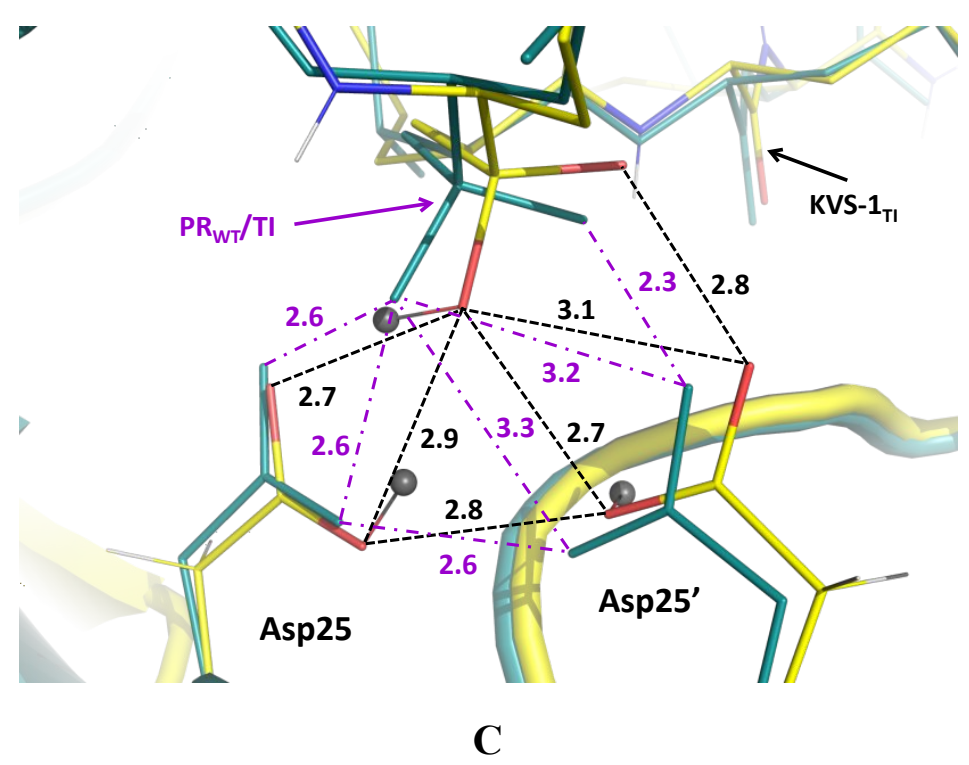


Figure S3. (A) Joint XN structure (yellow carbon atoms), superposed with unliganded HIV-1 PR (PDB ID 1LV1, green sticks) with r.m.s.d. of $0.4 \AA$ on the main chain atoms, shows catalytic water (green sphere) is located $0.7 \AA$ from hydroxyl of TI. The flap waters are $0.8 \AA$ apart. Other residues of KVS-1 ${ }_{\mathrm{TI}}$ are omitted for clarity. (B) Superposition of $\mathrm{PR}_{\mathrm{TM}} / \mathrm{KVS}-1_{\mathrm{TI}}$ (yellow and green carbon atoms) and $\mathrm{PR}_{\mathrm{I} 54 \mathrm{~V}} / \mathrm{TI}$ (grey carbon atoms, PDB ID 3B80) complexes showing non-covalent interactions within the catalytic sites. (C) Superposition of $\mathrm{PR}_{\mathrm{TM}} / \mathrm{KVS}-1_{\mathrm{TI}}$ (yellow carbon atoms) and $\mathrm{PR}_{\mathrm{WT}} / \mathrm{TI}$ (teal atoms, PDB ID 3B7V) complexes showing non-covalent interactions within the catalytic sites. O...O distances are in $\AA$. 


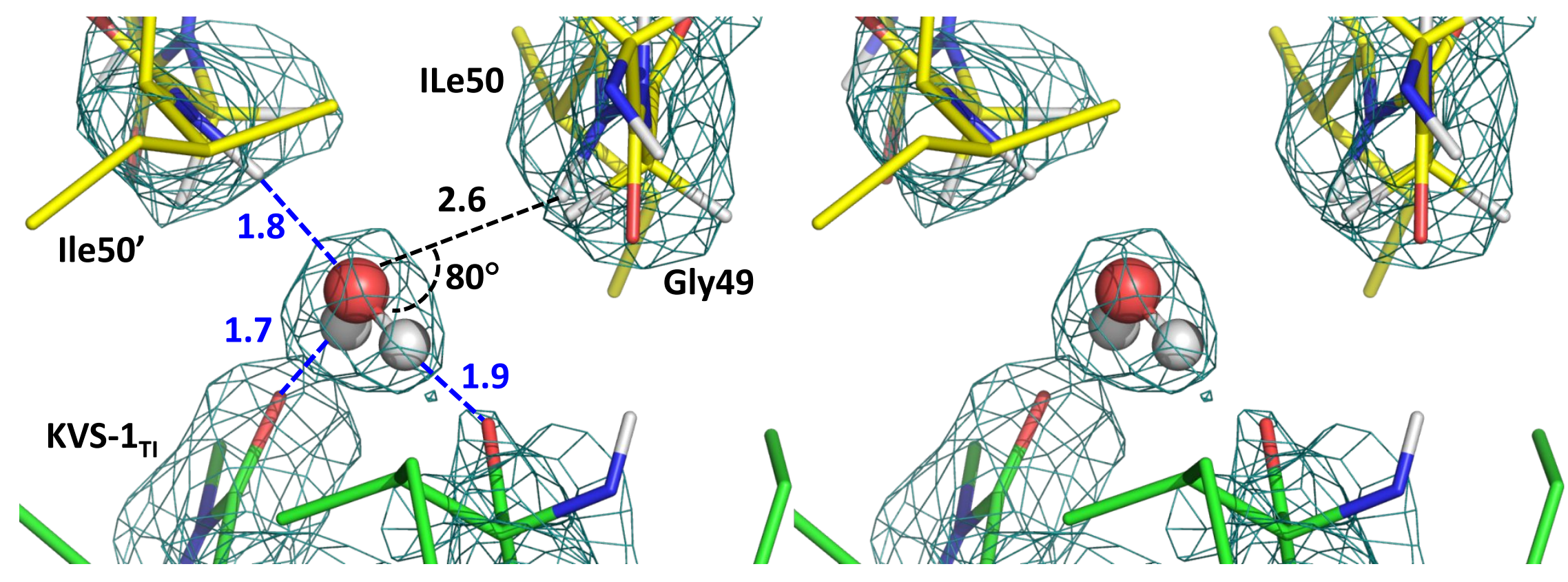

Figure S4. Figure 5B in crossed-eye stereo mode. 


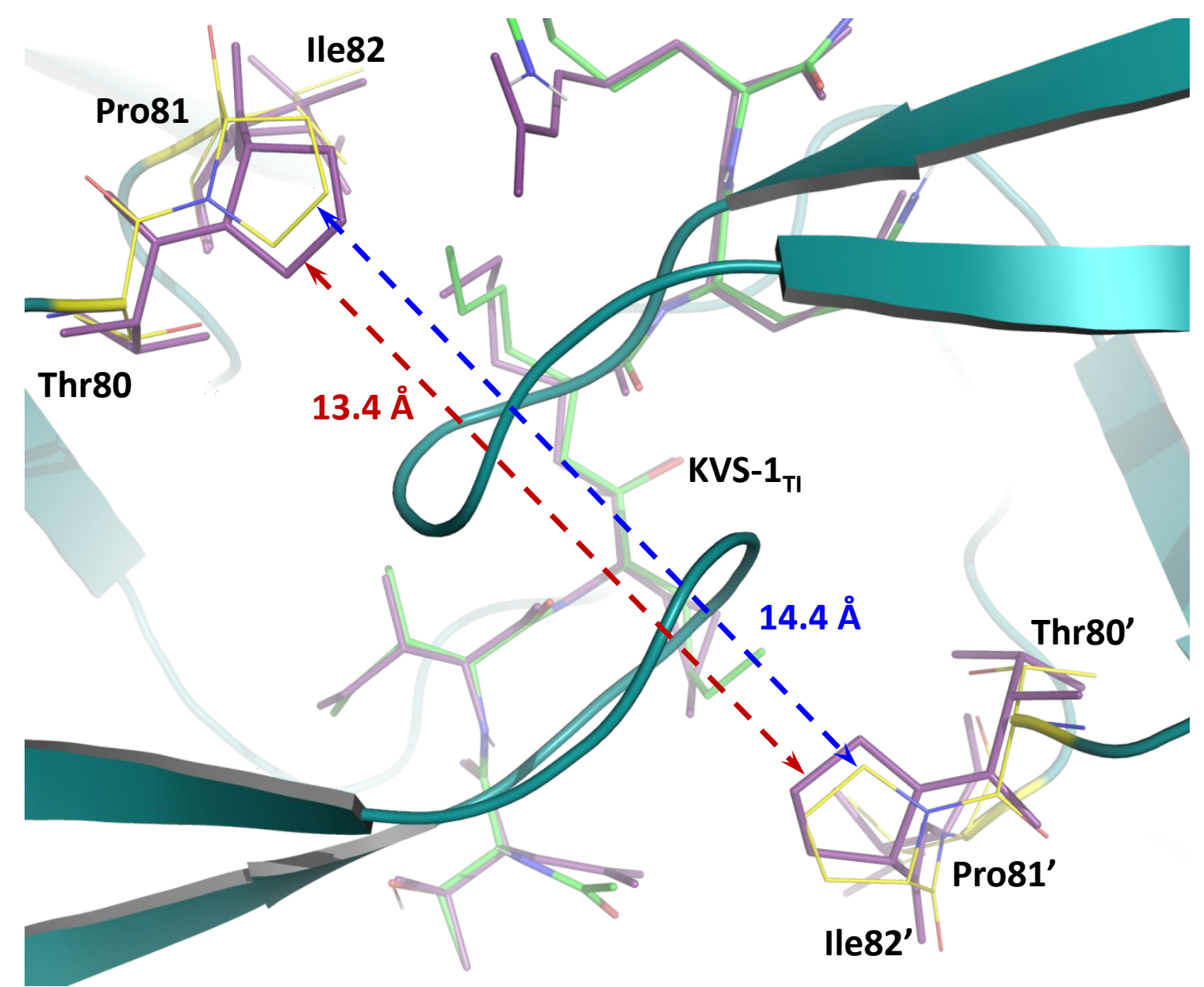

Figure S5. Superposition of room temperature joint XN (yellow and green carbon atoms) and low temperature X-ray (violet) structures of the $\mathrm{PR}_{\mathrm{TM}} / \mathrm{KVS}-1_{\mathrm{TI}}$ complex demonstrating a significant shift in the positions of the residues in the 80's loops. 


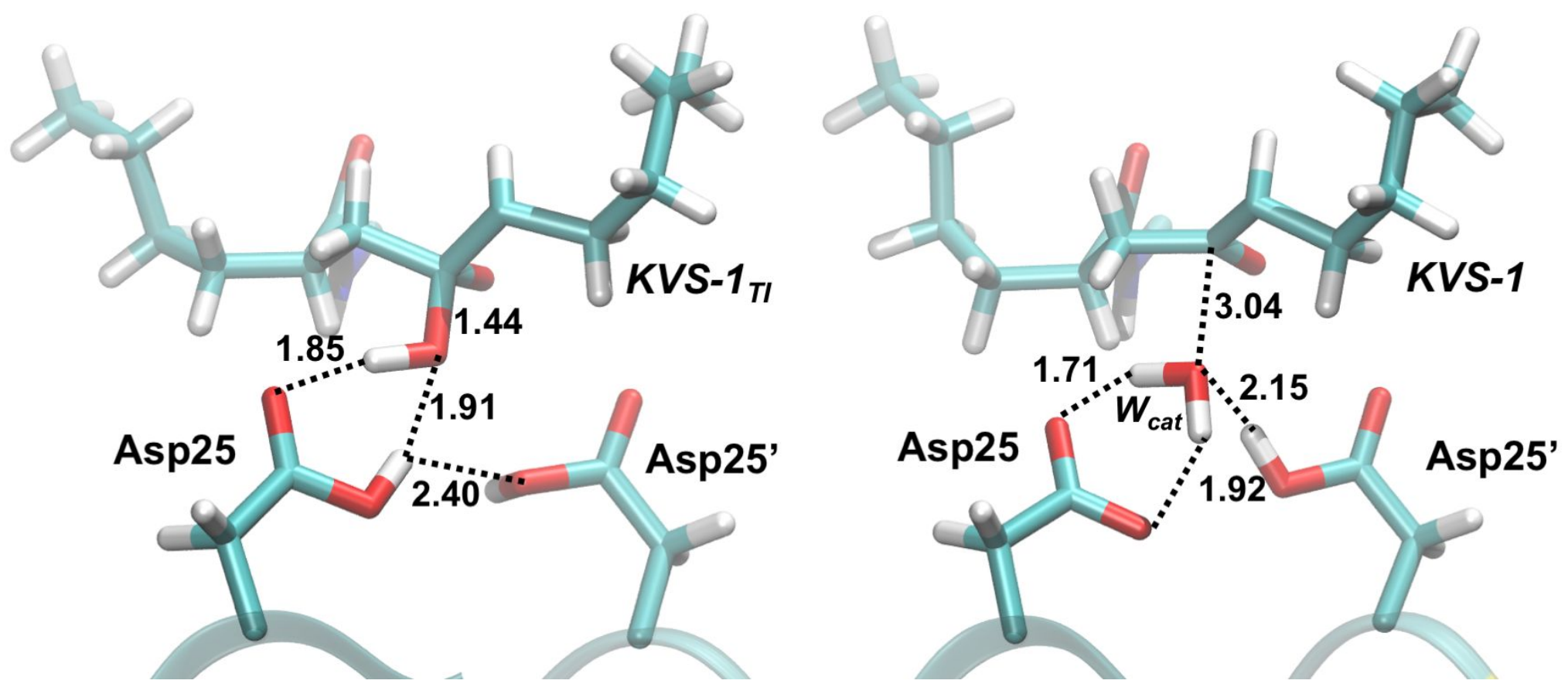

Figure S6. Catalytic site of the $\mathrm{PR}_{\mathrm{TM}} / \mathrm{KVS}-1_{\mathrm{TI}}$ complex: (left) starting geometry before $\mathrm{QM} / \mathrm{MM}$ optimization, and (right) $\mathrm{QM} / \mathrm{MM}$ optimized geometry revealing reactants, KVS-1 and the lytic water molecule $\left(\mathrm{W}_{\text {cat }}\right)$ are generated. 


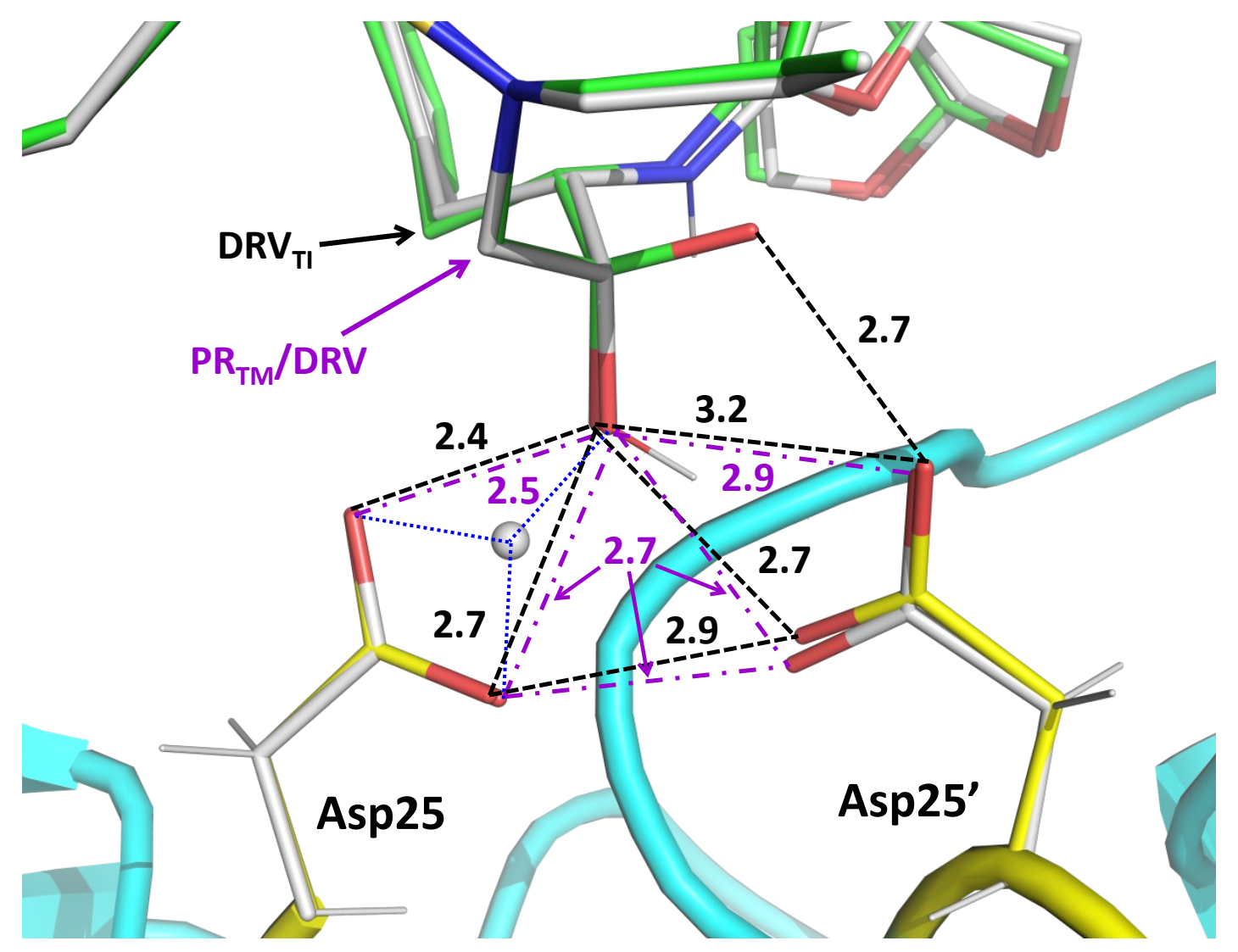

Figure S7. Superposition of $\mathrm{PR}_{\mathrm{TM}} / \mathrm{DRV}_{\mathrm{TI}}$ (yellow carbon atoms) and $\mathrm{PR}_{\mathrm{TM}} / \mathrm{DRV}$ (grey carbon atoms, $\mathrm{PDB}$ ID 5E5J) complexes showing non-covalent interactions within the catalytic sites. O...O distances are in $\AA$. The low-barrier hydrogen bond formed between Asp 25 and DRV hydroxy group is shown as blue dotted lines, with the $\mathrm{D}$ atom shown as grey sphere. 

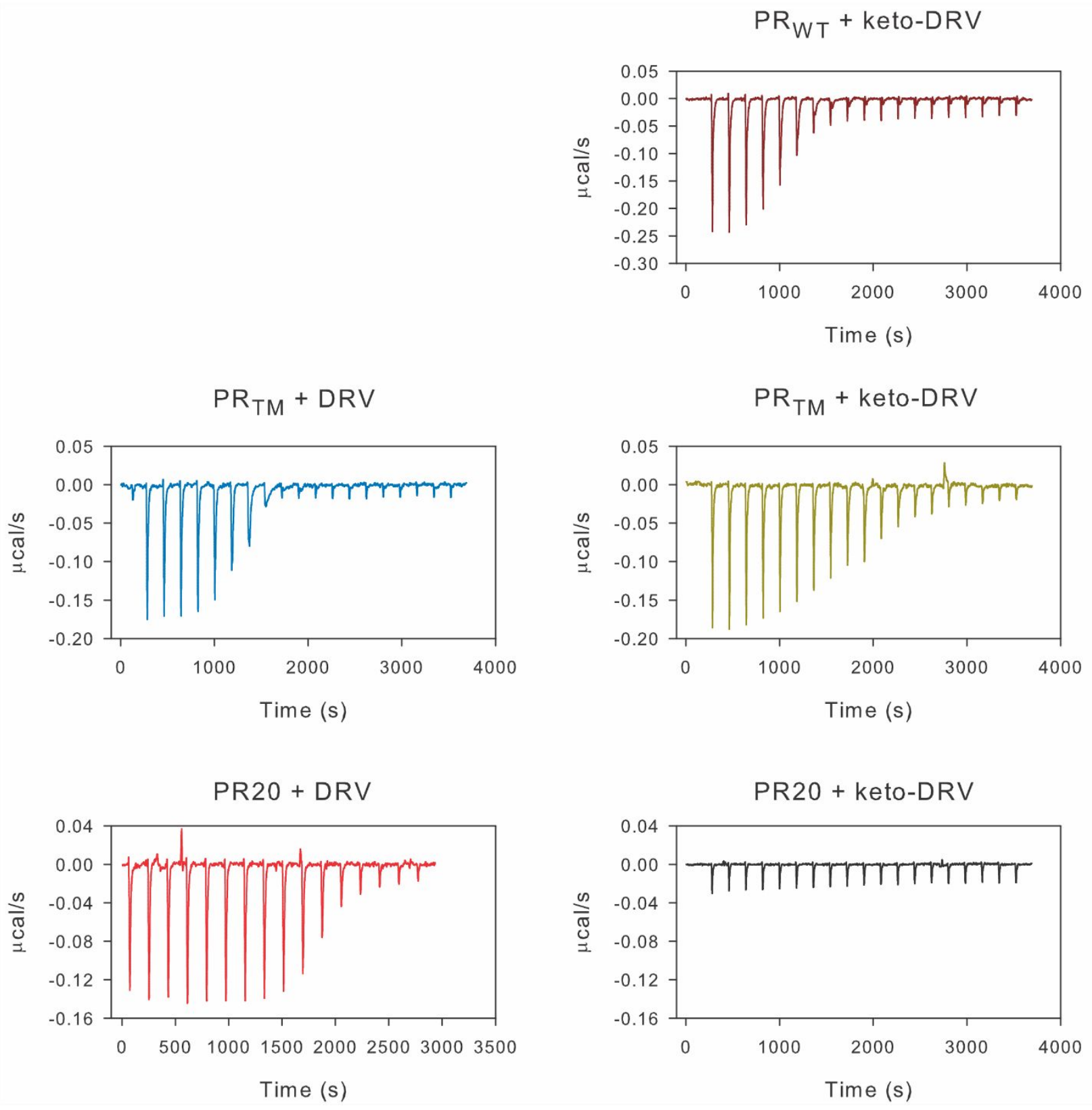

Figure S8. Raw ITC plots of inhibitor binding to protease shown in Figure 8 and Table 1. 

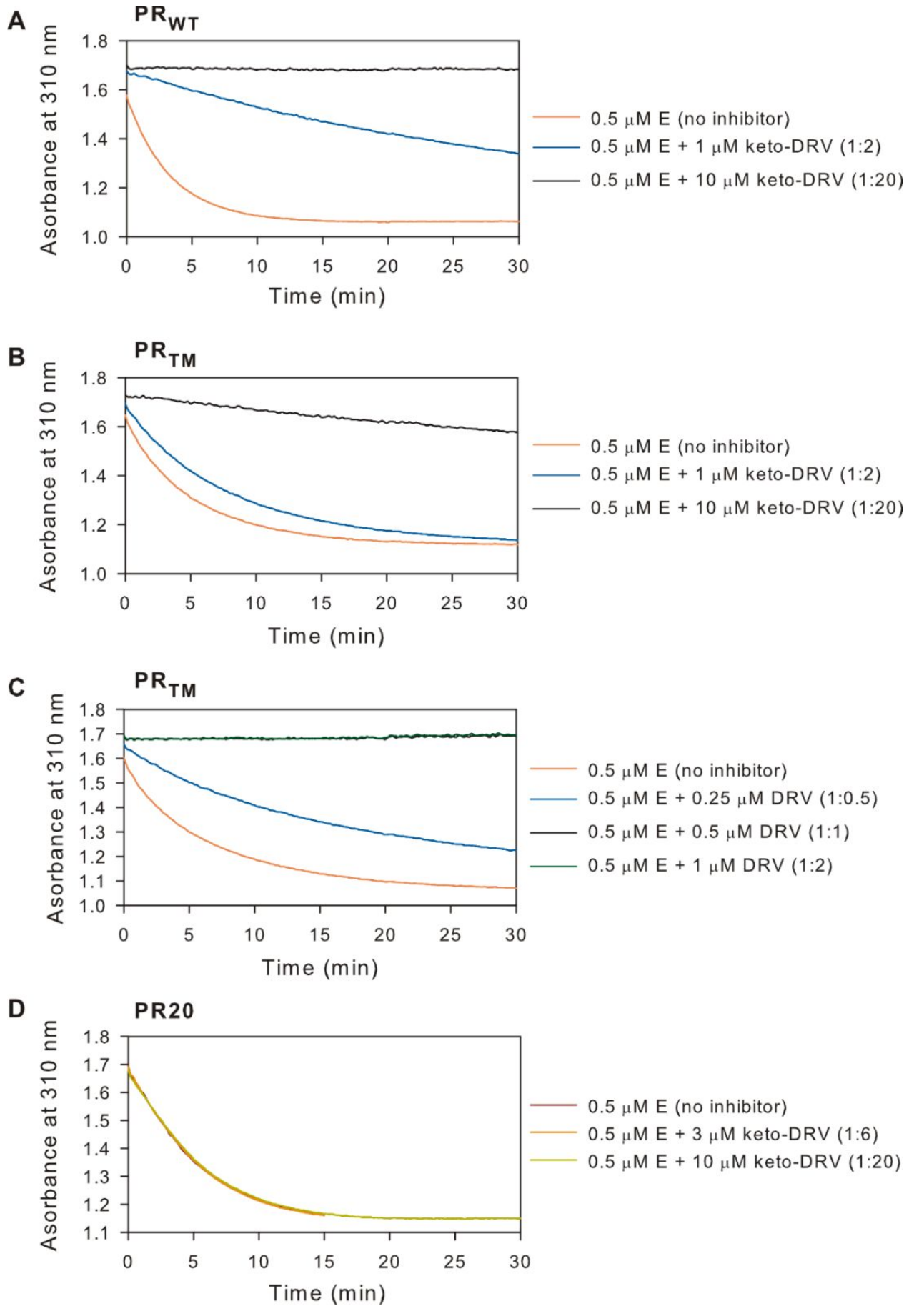

Figure S9. Hydrolysis of the chromogenic substrate catalyzed by the mature PR variants for (A, B) $\mathrm{PR}_{\mathrm{WT}}$ or $\mathrm{PR}_{\mathrm{TM}}+$ no inhibitor and + keto-DRV, (C) $\mathrm{PR}_{\mathrm{TM}}+$ no inhibitor and + DRV, (D) PR20 + no inhibitor and + keto-DRV. Traces follow the hydrolysis of the substrate accompanied by the decrease in absorbance at $310 \mathrm{~nm}$. E denotes enzyme. 


\section{REFERENCES}

1 Brooks, B. R.; Brooks 3d, C. L.; Mackerell Jr., A. D.; Nilsson,, L.; Petrella, R. J.; Roux, B.; Won, Y.; Archontis, G.; Bartels, C.; Boresch, S.; Caflisch, A.; Caves, L.; Cui, Q.; Dinner, A. R.; Feig, M.; Fischer, S.; Gao, J.; Hodoscek, M.; Im, W.; Kuczera, K.; Lazaridis, T.; Ma, J.; Ovchinnikov, V.; Paci, E.; Pastor, R. W.; Post, C. B.; Pu, J. Z.; Schaefer, M.; Tidor, B.; Venable, R. M.; Woodcock, H. L.; Wu, X.; Yang, W.; York, D. M.; Karplus, M. CHARMM: the Biomolecular Simulation Program. J. Comput. Chem. 2009, 30, 15451614.

2 Mackerell, A. D. Empirical Force Fields for Biological Macromolecules: Overview and Issues. J. Comput. Chem. 2004, 25, 1584-1604.

3 Mackerell, A. D.; Feig, M.; Brooks, C. L. Improved Treatment of the Protein Backbone in Empirical Force Fields. J. Am. Chem. Soc. 2004, 126, 698-699.

4 Vanommeslaeghe, K.; Hatcher, E.; Acharya, C.; Kundu, S.; Zhong, S.; Shim, J.; Darian, E.; Guvench, O.; Lopes, P.; Vorobyov, I.; Mackerell, A. D. CHARMM General Force Field: A Force Field for Drug-Like Molecules Compatible with the CHARMM All-Atom Additive Biological Force Fields. J. Comput. Chem. 2009, 31, 671-690.

5 Field, M. The pDynamo Program for Molecular Simulations Using Hybrid Quantum Chemical and Molecular Mechanical Potentials. J. Chem. Theory Comput. 2008, 4, 11511161.

6 Neese, F. The ORCA Program System. WIREs Comput. Mol. Sci. 2012, 2, 73-78.

7 Becke, A. D. Density-Functional Exchange-Energy Approximation with Correct Asymptotic Behavior. Phys. Rev. A Gen. Phys. 1988, 38, 3098-3100.

8 Lee, C.; Yang, W.; Parr, R. G. Development of the Colle-Salvetti Correlation-Energy Formula into a Functional of the Electron Density. Phys. Rev. B Condens. Matter. 1988, 37, 785-789 (1988).

9 Perdew, J. P.; Burke, K.; Ernzerhof, M. Generalized Gradient Approximation Made Simple. Phys. Rev. Lett. 1996, 77, 3865-3868.

10 Perdew, J. P.; Emzerhof, M.; Burke, K. Rationale for Mixing Exact Exchange with Density Functional Approximations. J. Chem. Phys. 1996, 105, 9982-9985.

11 Adamo, C.; Barone, V. Toward Reliable Density Functional Methods without Adjustable Parameters: The PBE0 Model. J. Chem. Phys. 1999, 110, 6158-6170.

12 Zhao, Y.; Truhlar, D. G. The M06 Suite of Density Functionals for Main Group Thermochemistry, Thermochemical Kinetics, Noncovalent Interactions, Excited States, and Transition Elements: Two New Functionals and Systematic Testing of Four M06-Class Functionals and 12 Other Functionals. Theoret. Chem. Acc. 2008, 120, 215-241.

13 Zhao, Y.; Truhlar, D. G. Density Functionals with Broad Applicability in Chemistry. Acc. Chem. Res. 2008, 41, 157-167.

14 Becke, A. D. Density-Functional Thermochemistry .3. The Role of Exact Exchange. J. Chem. Phys. 1993, 98, 5648-5652.

15 Vosko, S. H.; Wilk, L.; Nusair, M. Accurate Spin-Dependent Electron Liquid Correlation Energies for Local Spin-Density Calculations - a Critical Analysis. Can. J. Phys. 1980, 58, 1200-1211.

16 Chai, J. D.; Head-Gordon, M. Systematic Optimization of Long-Range Corrected Hybrid Density Functionals. J. Chem. Phys. 2008, 128, 084106. 
17 Chai, J. D.; Head-Gordon, M. Long-Range Corrected Hybrid Density Functionals with Damped Atom-Atom Dispersion Corrections. Phys. Chem. Chem. Phys. 2008, 10, 66156620.

18 Grimme, S. Density Functional Theory with London Dispersion Corrections. Wiley Interdiscip. Rev.: Comput. Mol. Sci. 2011, 1, 211-228.

19 Grimme, S.; Ehrlich, S.; Goerigk, L. Effect of the Damping Function in Dispersion Corrected Density Functional Theory. J. Comput. Chem. 2011, 32, 1456-1465.

20 Grimme, S. Improved Second-Order Moller-Plesset Perturbation Theory by Separate Scaling of Parallel- and Antiparallel-Spin Pair Correlation Energies. J. Chem. Phys. 2003, 118, 9095-9102.

21 Karplus P.A., Diederichs K. Linking crystallographic model and data quality. Science 336, 1030-1033. 